1 King's College Hospital NHS Trust, London SE5 9RS, UK

2 Cicely Saunders Institute, King's College London, London SE5 9PJ, UK Correspondence to: R Ting

Cite this as: $B M J 2020 ; 370: \mathrm{m} 2710$ http://dx.doi.org/10.1136/bmj.m2710

Published: 14 July 2020

\title{
Palliative care for patients with severe covid-19
}

\author{
Ruth Ting, ${ }^{1}$ Polly Edmonds, ${ }^{1}$ Irene J Higginson, ${ }^{1,2}$ Katherine E Sleeman ${ }^{1}, 2$
}

\section{What you need to know}

- Many patients with severe covid-19 experience distressing symptoms, including breathlessness and agitation. Palliation of suffering is an important part of care irrespective of prognosis

- Patients with severe covid-19 may deteriorate rapidly. It is therefore useful to have a strategy in place for managing deterioration and potential death (for those not suitable for escalation to intensive care), which runs alongside the acute medical management plan

- Clear and timely communication with the patient (if they are able) and their carers is essential. Conveying hope that treatments will help needs to be sensitively balanced with explicit acknowledgement that patients are sick enough to die

"To cure sometimes, to relieve often, and to comfort always."

variously attributed to William Osler, Edward Trudeau, and Hippocrates

On 12 March 2020 covid-19 was declared a pandemic by the World Health Organisation. Approximately one in five people with covid-19 aged over 80 require hospitalisation. ${ }^{1}$ An observational study of 20133 people with covid-19 requiring hospitalisation in the UK found that $26 \%$ died, $41 \%$ were discharged alive, while $34 \%$ remained inpatients at the time of reporting. ${ }^{2}$ The case fatality rate varies worldwide, with the risk of death higher among people who are older, male, multimorbid, of black, Asian, and minority ethnicity, and from areas of higher deprivation. ${ }^{3-5}$

Palliative care is commonly misunderstood as only being relevant for people who are dying. However, the relief of suffering, through provision of holistic and compassionate care, is an essential component of care for all patients with life threatening illness. This article outlines the palliative approach to the management of patients with severe covid-19 in hospital and community settings, focusing on the management of distressing symptoms, planning ahead, communicating with patients and their families, and grief and bereavement. The clinical triage of critically ill patients with severe covid-19 (to determine those most likely to benefit from escalation to high dependency or intensive care) is outside of the scope of this article.

\section{Symptoms in patients with severe covid-19}

Symptoms can escalate rapidly among patients with severe covid-19. An early case series from Wuhan in China of hospital presentations found that the median time from first symptom to breathlessness was five days, and to acute respiratory distress syndrome (ARDS) was eight days. ${ }^{6}$ A report of 6801 patients who died with covid-19 in Italy found that the median time from onset of symptoms to hospitalisation was five days, and from onset of symptoms to death was nine days. ${ }^{7}$ Therefore, an anticipatory approach to symptom management for people with severe covid-19 is key. ${ }^{8}$

Among decedents and survivors with covid-19, breathlessness, cough, and fatigue are the most common symptoms. ${ }^{9}$ A case series of 101 patients with severe covid-19 who were referred to three hospital palliative care teams in London, UK, found breathlessness and agitation were the most common symptoms, alongside drowsiness and delirium. ${ }^{10}$

\section{How can breathlessness in severe covid-19 be managed?}

\section{Opioids}

The mainstay of pharmacological management of severe breathlessness is opioids, with morphine being the opioid of choice in the absence of renal impairment. ${ }^{112}$ Opioids should be considered in patients who are severely breathless at rest or on minimal exertion. If the patient is able to take oral medication, immediate release oral morphine can be used (such as $2.5 \mathrm{mg}$ every 4 hours). If the patient is unable to swallow or is drowsy or unconscious morphine can be given parenterally to relieve breathlessness. If the patient remains breathless despite the use of opioids, involvement of a palliative care team should be considered.

The use of continuous parenteral infusions can be useful to ensure a consistent background dose of opioid and enable titration according to symptom severity. An early case series of 101 hospitalised patients with covid-19 who were referred to palliative care found that opioids were usually effective for palliation of breathlessness, with a median dose of $10 \mathrm{mg}$ (range 5-30 mg) subcutaneous morphine over 24 hours. ${ }^{10}$ A retrospective audit of 36 hospitalised patients with covid-19 who died outside critical care found that 26 of them had a subcutaneous infusion at the time of death, and the total mean opioid dose (subcutaneous morphine equivalent) during the final 24 hours was $16 \mathrm{mg} \cdot{ }^{13}$

In people with severe covid-19, who may deteriorate rapidly, there should be a low threshold for the use of parenteral infusions so that patients do not die before their symptoms can be controlled. Continuous parenteral infusions have the advantage of reducing the requirement for frequent "as needed" doses. Some symptomatic patients with severe breathlessness will still require "as needed" doses, and these should remain available to the patient to support symptom management. Use of an alternative opioid, such as fentanyl or alfentanil, should be considered in people with significant renal impairment, taking care to achieve equianalgesic starting doses. 
Non-pharmacological management of breathlessness includes staff having a calm and reassuring manner and using cool wipes on the face, ensuring these are safely disposed of after each use. The use of a handheld fan is not recommended in covid-19 because of the theoretical risk of increasing droplet spread. While use of the prone position can improve mortality among people with severe covid-19 related ARDS, ${ }^{14}$ there are no published data on the effectiveness of proning for palliation. In one study of patients with covid-19 and hypoxaemic respiratory failure who were managed outside of intensive care, $63 \%$ were able to tolerate proning for more than three hours, although the benefit was unclear. ${ }^{15}$ Patients who are able to adopt the prone position themselves should be encouraged to try it for relief of breathlessness.

\section{What about oxygen therapy?}

Oxygen therapy may help relieve breathlessness in severe hypoxaemia, and many patients with severe covid-19 in hospital will be prescribed oxygen. A recent rapid review found no evidence to support the use of oxygen for breathlessness among people with covid-19 in the absence of hypoxaemia. ${ }^{16}$ For patients dying from covid-19, the priority is to treat the symptom of breathlessness rather than oxygen saturation levels. The simplest way to assess for severity of breathlessness is to ask the patient if they are feeling breathless while at rest and observe for signs of increased work of breathing (inability to complete sentences, use of accessory muscles, and raised respiratory rate). It should be noted that some patients with hypoxaemia have minimal breathlessness. In dying patients, if symptoms are well managed, oxygen therapy may be titrated down while carefully monitoring symptoms. There should be an individualised approach, and some patients who are hypoxic may gain symptomatic benefit from oxygen therapy. If so, nasal cannulae may be more comfortable than a face mask. For people dying while still receiving non-invasive ventilation support, specialist advice should be sought.

\section{How can agitation and anxiety be managed in severe covid-19?}

Agitation and anxiety are common among people with severe covid-19 and are managed with a combination of pharmacological and non-pharmacological treatments. For people with severe agitation, especially those who may be approaching the end of life, benzodiazepines can be given, alone or in combination with opioids if needed. ${ }^{17}$ As with opioids, titration of the dose of benzodiazepine may be needed to achieve good symptom relief. If there is an element of delirium (such as disorientation to time, place, or person) contributing to agitation or anxiety, an antipsychotic such as haloperidol may be needed instead of or in addition to benzodiazepines.

Patients with severe covid-19 may experience anxiety and agitation due to a combination of physically distressing symptoms, isolation and separation from loved ones, and the challenges of being cared for by professionals dressed in personal protective equipment (PPE). Distress may be compounded by anxiety concerning the diagnosis and fear that they may die. It is important to acknowledge and address these factors individually. Innovations to alleviate distress include the use of video calls with families, and healthcare professionals writing their own names or pinning photos of their faces onto their PPE to humanise themselves when interacting with patients. ${ }^{18}$ If a patient with severe covid-19 expresses a fear of dying: (a) reassure them that they are receiving treatments that aim to support their bodies to recover from the effects of the virus, but be honest that there is still a worry they are very unwell and could die; (b) reassure them that treatments are available to ensure that they are comfortable; and $(c)$ ask them what is important to them right now and if there is anyone they would like you to speak to about their situation.

\section{Specific factors to consider in community settings}

For patients who prefer to be cared for in a community setting (in their own home or a care home) rather than be admitted to hospital, practical challenges include access to PPE and oxygen, and the availability of trained healthcare professionals to administer medication parenterally if needed. In the UK, guidance is available to support community services ${ }^{19}$ and carers $^{20}$ of patients with covid-19.

For patients who are unable to take medication orally, parenteral medication is usually administered by community nursing staff. In circumstances where professionals are not available, family members may be trained to administer medicines. While still uncommon in the UK, this is common practice in other settings, such as in rural Australia. ${ }^{21}$

\section{Preparing an urgent care plan in case of deterioration and death}

Covid-19 can progress rapidly, and severely affected patients are likely to lose their capacity to make decisions. The rapidity with which patients can deteriorate means that for many it is appropriate to have a holistic "urgent care plan" in place in the event of acute deterioration. This should run alongside the acute medical management plan and encompass anticipatory medication for symptom management as well as recognition of the patient's wishes and preferences (see box 1). ${ }^{22}$ To make a plan consistent with a patient's wishes, it is crucial to have a discussion with them as early as possible, even as early as when they first present to hospital or are seen in the community setting with likely covid-19. If the patient no longer has capacity to make their thoughts and wishes known, healthcare professionals should explore previously expressed preferences from those who know them well, to inform decision making in their best interests.

Box 1: Key areas to explore when developing holistic urgent care plans for patients at risk of severe covid-19

- Find out if the patient has ever expressed or formally documented preferences for managing a life threatening condition. This includes whether they have legally appointed a surrogate decision-maker or created a legally binding document specifying treatment preferences (such as an advance decision to refuse treatment)

- Explain the medical treatment escalation plan, including whether cardiopulmonary resuscitation, respiratory support, and other organ support in intensive care are medically appropriate

- Explain that, alongside the treatment escalation plan, there will be a plan in place for symptom management to ensure the patient's comfort whatever the outcome may be

- Ask: "Knowing what you know now of your situation, is there anything else important to you at this time?"

- Ask: "Who should we contact in the event that you are unwell?" Ensure that their contact details are recorded clearly

\section{What is the best way to communicate with patients and families?}

It is important to communicate with patients and relatives using clear language and in a timely manner, if needed several times a day, taking into account the urgency of conveying important updates if the patient is deteriorating. ${ }^{23}$ 
Communication is more challenging during the covid-19 pandemic as health professionals will be speaking to patients under PPE, which muffles voices and obscures non-verbal cues; this can be compounded if patients are supported by loud equipment such as a continuous positive airway pressure machines or high flow oxygen. Difficulty communicating can become more pronounced with patients who are delirious or who have hearing or sight impairment.

Communication with relatives during the covid-19 pandemic is likely to be by phone or video call. Emerging evidence supports use of video consultations with patients and family members as an effective, accessible, and acceptable method of communication. ${ }^{2425}$ Communicating via video can enhance the "therapeutic presence" of the healthcare professional compared with a phone call. ${ }^{26}$

Communication is also challenging for people with covid-19 because the outcome is uncertain. Rapid deterioration for some patients means that health professionals may not have time to form a relationship with the patient and their families before conveying bad news. Balancing hopes and fears requires a combination of honesty and compassion. Hope-worry statements, which balance hope that the patient will recover with expression of worry that they may not, can be useful (box 2). The phrase "sick enough to die" has been suggested as a way of conveying the possibility that a patient may deteriorate and die while acknowledging uncertainty. ${ }^{27}$ Honest conversations delivered well provide patients and families with the choice to use that information, should they wish, to organise what is important in their lives and to perhaps say what they need to say in the event that they do not recover.

Box 2: Phrases that might be helpful when communicating with patients with severe covid-19 and their families and friends

- Hope-worry statements-“We are doing all we can. We hope you/your relative will start to respond, but I am worried you/they may continue to deteriorate in spite of our best medical treatments."

- Sick enough to die-“I'm afraid there has been a sudden/ongoing deterioration, and you are/your relative is now sick enough to die."

- Advance care planning-"We are hoping for the best, but it would be wise to start planning for the worst. Knowing the full picture now, what is important to you/your loved ones at this time?"

- Comfort and reassurance-"We are doing all we can, and, whichever path this takes, we will do our best to ensure you are/your relative is comfortable."

- Supportive or empathic statements-“I'm so sorry that we're talking about your loved one by phone and not face-to-face." "This must be such a difficult thing to hear by phone." "We are constantly checking your loved one, and we will ensure they get what they need."

Resources for patients and carers to help them understand what to expect when they have severe covid-19 may be useful (for example, the European Lung Foundation has produced information leaflets for patients admitted to hospital with covid-19 (https://www.europeanlung.org/en/news-and-events/news/covid-19-factsheets)).

\section{Grief and bereavement}

Grief is a natural human emotion in response to significant loss. Deaths due to covid-19 may be associated with risk factors that can lead to poor bereavement outcomes among relatives and distress in frontline staff. ${ }^{28}$ During the covid-19 pandemic grief may be significantly affected by other losses due to social distancing restrictions, such as not being with a loved one as they are dying, not being able to attend a funeral, and not being able to seek comfort in the arms of wider friends and family while grieving. ${ }^{29}$ Additional risk factors for poor bereavement outcomes and complicated grief in covid-19 include severe breathlessness, patient isolation, and disruption to the relative's social support networks. ${ }^{30}$ Ways to mitigate these include:

- Proactive, sensitive, and regular communication with carers alongside accurate information provision

- Enabling relatives to say goodbye in person where possible and supporting virtual communication

- Provision of excellent palliation of symptoms

- Provision of emotional and spiritual support before death

- Signposting to bereavement support services and encouraging relatives to access these in a timely fashion.

\section{Looking after yourself and each other at work}

The pandemic has been challenging for individuals and communities all over the world.

It is entirely normal for healthcare professionals to have an emotional reaction to their experiences at work, especially when there has been an increase in deaths in their clinical areas, and this may lead to anxiety for themselves and their colleagues. It is important to acknowledge these feelings and encourage colleagues to do the same. People may find applying strategies that have been effective in the past to cope with challenging times to be helpful; some will wish to seek additional help and guidance, which is available from a range of resources, including employers and primary care, chaplaincy and faith groups, and palliative care.

\section{Additional education resources}

- Association for Palliative Medicine, UK. COVID-19 and palliative, end of life and bereavement care in secondary care. https://apmonline.org/wp-content/uploads/2020/04/COVID-19-and-Palliative-Endof-Life-and-Bereavement-Care-20-April-2020-2.pdf

- National Institute for Health and Care Excellence. COVID-19 rapid guideline: managing symptoms (including at the end of life) in the community (NICE guideline NG163). 2020. https://www.nice.org.uk/guidance/ng163

- E-Learning for Healthcare. Coronavirus (COVID-19) resources. https://portal.e-lfh.org.uk/Catalogue/Index?Hierarchyld=0_45016\&programmeld $=45016$

Includes resources for staff working in acute hospital setting, critical care and primary care and community settings

- Cicely Saunders Institute, King's College London. COVID-19 resources. https://www.kcl.ac.uk/cicelysaunders/resources/links

\section{Information resources for patients}

- European Lung Foundation. COVID-19 hospital factsheets: Factsheets for people hospitalised with COVID-19 and family/friends.

https://www.europeanlung.org/en/news-and-events/news/covid19-factsheets

- British Psychological Society. Coping with death and grief during Covid-19. https://www.bps.org.uk/coronavirus-resources/public/coping-death-and-grief

- British Psychological Society. Talking to children about coronavirus. https://www.bps.org.uk/coronavirus-resources/public/talking-children-about-coronavirus

- Hospice UK. Caring for your dying relative at home with COVID-19. https://www.hospiceuk.org/docs/default-source/echo/covid-19echo/covid-19_care-at-home_guide_final.pdf?sfvrsn=4 
- Helix Centre. Coronavirus worst case scenario planning. Educational video series: 6 . How to have the conversation about the worst case [with your patient]. https://conversation.helixcentre.com/

- One of a series of six videos by Justin Avery, GP and specialist in palliative care

\section{Sources and selection criteria}

We searched PubMed using terms "palliative" and "COVID" to identify published data on palliative care for people with severe covid-19, and used our clinical experience of looking after people with severe covid-19 to supplement this.

\section{How patients were involved in the creation of this article}

The article was reviewed by members of the Patient and Public Involvement group at the Cicely Saunders Institute of Palliative Care, Policy and Rehabilitation, London. Changes were made on this feedback, in particular to the urgent care plan and communication sections.

Contributors: All authors contributed to drafts and revisions of the manuscript and approved the final version for publication. RT is the guarantor.

Competing interests: We have read and understood BMJ policy on declaration of interests and have no relevant interests to declare.

Funding: KES is funded by a National Institute of Health Research (NIHR) Clinician Scientist Fellowship (CS-2015-15-005), IJH is an NIHR Senior Investigator Emeritus and is supported by the NIHR Applied Research Collaboration South London (NIHR ARC South London) at King's College Hospital NHS Foundation Trust. The views expressed are those of the authors and not necessarily those of the NHS, the NIHR, or the Department of Health and Social Care.

Provenance and peer review: Commissioned; externally peer reviewed.

1 Verity R, Okell LC, Dorigatti I, etal. Estimates of the severity of coronavirus disease 2019: a model-based analysis. Lancet Infect Dis 2020;20:669-77. doi: 10.1016/S1473-3099(20)30243-7 pmid: 32240634

2 Docherty AB, Harrison EM, Green CA, etallSARIC4C investigators. Features of 20133 UK patients in hospital with covid-19 using the ISARIC WHO Clinical Characterisation Protocol: prospective observational cohort study. BMJ2020;369:m1985. doi: 10.1136/bmj.m1985 pmid: 32444460

3 Guan WJ, Liang WH, Zhao Y, etalChina Medical Treatment Expert Group for COVID-19. Comorbidity and its impact on 1590 patients with COVID-19 in China: a nationwide analysis. Eur Respir J 2020;55:. doi: 10.1183/13993003.00547-2020 pmid: 32217650

4 Zhou F, Yu T, Du R, etal. Clinical course and risk factors for mortality of adult inpatients with COVID-19 in Wuhan, China: a retrospective cohort study. Lancet 2020;395:1054-62. doi: 10.1016/S0140-6736(20)30566-3 pmid: 32171076

5 Williamson EJ, Walker AJ, Bhaskaran KJ, etal. OpenSAFELY: factors associated with COVID-19 death in 17 million patients. Nature 2020. doi: 10.1038/s41586-020-2521-4. pmid: 32640463

6 Wang D, Hu B, Hu C, etal. Clinical characteristics of 138 hospitalized patients with 2019 novel coronavirus-infected pneumonia in Wuhan, China. JAMA 2020;323:1061-9. doi: 10.1001/jama.2020.1585 pmid: 32031570

7 COVID-19 Surveillance Group. Characteristics of COVID-19 patients dying in Italy. Report based on available data on March 26th, 2020. https://www.epicentro.iss.it/coronavirus/bollettino/ReportCOVID-2019_26_marzo_eng.pdf.

8 Bajwah S, Wilcock A, Towers R, etal. Managing the supportive care needs of those affected by COVID-19. Eur Respir J2020;55:. doi: 10.1183/13993003.00815-2020 pmid: 32269090

9 Keeley P, Buchanan D, Carolan C, Pivodic L, Tavabie S, Noble S. Symptom burden and clinical profile of COVID-19 deaths: a rapid systematic review and evidence summary. BMJ Support Palliat Care 2020; . doi: 10.1136/bmjspcare-2020-002368 pmid: 32467101

10 Lovell N, Maddocks M, Etkind SN, etal. Characteristics, symptom management and outcomes of 101 patients with COVID-19 referred for hospital palliative care. J Pain Symptom Manage 2020;60:e77-81. doi: 10.1016/j.jpainsymman.2020.04.015 pmid: 32325167

11 Ekström M, Nilsson F, Abernethy AA, Currow DC. Effects of opioids on breathlessness and exercise capacity in chronic obstructive pulmonary disease. A systematic review. Ann Am Thorac Soc 2015;12:1079-92. doi: 10.1513/AnnalsATS.201501-0340C pmid: 25803110

12 Ekström M, Bajwah S, Bland JM, Currow DC, Hussain J, Johnson MJ. One evidence base; three stories: do opioids relieve chronic breathlessness? Thorax 2018;73:88-90. doi: 10.1136/thoraxjnl-2016-209868 pmid: 28377491

13 Turner J, Eliot Hodgson L, Leckie T, Eade L, Ford-Dunn S. A dual-centre observational review of hospital based palliative care in patients dying with COVID-19. J Pain Symptom Manage 2020;. doi: 10.1016/j.jpainsymman.2020.04.031. pmid: 32387139

14 Ghelichkhani P, Esmaeili M. Prone position in management of COVID-19 patients; a commentary. Arch Acad Emerg Med 2020;8:.pmid: 32309812
15 Elharrar X, Trigui Y, Dols AM, etal. Use of prone positioning in nonintubated patients with COVID-19 and hypoxemic acute respiratory failure. JAMA 2020. doi: 10.1001/jama.2020.8255. pmid: 32412581

16 Allsop M, Ziegler L, Fu Y, Rudd S, Bennett MI; Oxford COVID-19 Evidence Service Team. Is oxygen an effective treatment option to alleviate the symptoms of breathlessness for patients dying with COVID-19 and what are the potential harms? CEBM, 2020. https://www.cebm.net/covid19/is-oxygen-an-effective-treatment-option-to-alleviate-the-symptoms-of-breathlessness-forpatients-dying-with-covid-19-and-what-are-the-potential-harms/.

17 Navigante AH, Cerchietti LC, Castro MA, Lutteral MA, Cabalar ME. Midazolam as adjunct therapy to morphine in the alleviation of severe dyspnea perception in patients with advanced cancer. Pain Symptom Manage2006;31:38-47. doi: 10.1016/j.jpainsymman.2005.06.009 pmid: 16442481

18 ihub Healthcare Improvement Scotland. People-led care: person-centred health and care. 2020 https://ihub.scot/improvement-programmes/people-led-care/person-centred-health-andcare/supporting-person-centred-care-in-covid-19-situations.

19 National Institute for Health and Care Excellence. COVID-19 rapid guideline: managing symptoms (including at the end of life) in the community (NICE guideline NG163). 2020. https://www.nice.org.uk/guidance/ng163.

20 Hospice UK. Caring for your dying relative at home with COVID-19. 2020. https://www.hospiceuk.org/docs/default-source/echo/covid-19-echo/covid-19_care-at-home_guide_final.pdf.

21 Bowers B, Pollock K, Barclay S. Administration of end-of-life drugs by family caregivers during covid-19 pandemic. BMJ2020;369:m1615. doi: 10.1136/bmj.m1615 pmid: 32332027

22 Curtis JR, Kross EK, Stapleton RD. The importance of addressing advance care planning and decisions about do-not-resuscitate orders during novel coronavirus 2019 (COVID-19). JAMA 2020; . doi: 10.1001/jama.2020.4894 pmid: 32219360

23 Hart JL, Turnbull AE, Oppenheim IM, Courtright KR. Family-centered care during the COVID-19 era. J Pain Symptom Manage 2020;. doi: 10.1016/j.jpainsymman.2020.04.017. pmid: 32333961

24 Sutherland AE, Stickland J, Wee B. Can video consultations replace face-to-face interviews? Palliative medicine and the Covid-19 pandemic: rapid review. BMJ Support Palliat Care 2020;. doi: 10.1136/bmjspcare-2020-002326 pmid: 32457086

25 Kuntz JG, Kavalieratos D, Esper GJ, Ogbu N, JrMitchell J, McLean Ellis C. Feasibility and Acceptability of Inpatient Palliative Care E-Family Meetings During COVID-19 Pandemic. J Pain Symptom Manage 2020;. doi: 10.1016/j.jpainsymman.2020.06.001. pmid: 32505643

26 Greenhalgh T, Koh GCH, Car J. Covid-19: a remote assessment in primary care. BMJ 2020;368:m1182. doi: 10.1136/bmj.m1182 pmid: 32213507

27 Mannix K. With the End in Mind. How to Live and Die Well . Collins, 2018.

28 Selman LE, Chao D, Sowden R, Marshall S, Chamberlain C, Koffman J. Bereavement Support on the Frontline of COVID-19: Recommendations for Hospital Clinicians. J Pain Symptom Manage 2020;. doi: 10.1016/j.jpainsymman.2020.04.024 pmid: 32376262

29 Mayland CR, Harding AJE, Preston N, Payne S. Supporting adults bereaved through COVID-19: a rapid review of the impact of previous pandemics on grief and bereavement. J Pain Symptom Manage 2020;. pmid: 32416233

30 Wallace CL, Wladkowski SP, Gibson A, White P. Grief during the COVID-19 pandemic: considerations for palliative care providers. J Pain Symptom Manage 2020;60:e70-6. doi: 10.1016/j.jpainsymman.2020.04.012 pmid: 32298748 